\title{
Sulfonylurea-resistant biotypes of Monochoria vaginalis generate higher ultraweak photon emissions than the susceptible ones
}

\section{$\operatorname{AUTHOR}(\mathrm{S}):$}

Inagaki, Hidehiro; Imaizumi, Toshiyuki; Wang, Guang-Xi; Tominaga, Tohru; Kato, Kimihiko; lyozumi, Hiroyuki; Nukui, Hideki

\section{CITATION:}

Inagaki, Hidehiro ... [et al]. Sulfonylurea-resistant biotypes of Monochoria vaginalis generate higher ultraweak photon emissions than the susceptible ones. Pesticide Biochemistry and Physiology 2009, 95(3): 117-120

\section{ISSUE DATE:}

2009-08

URL:

http://hdl.handle.net/2433/85405

\section{RIGHT:}

c 2009 Elsevier Inc. All rights reserved.; This is not the published version. Please cite only the published version.; この論文は出版社版でありませ ん。引用の際には出版社版をご確認ご利用ください。 

generate higher ultraweak photon emissions than the susceptible ones

4 Hidehiro Inagaki ${ }^{\text {a }}$, Toshiyuki Imaizumi ${ }^{b}$, Guang-Xi Wang ${ }^{\text {b,* }}$, Tohru Tominaga ${ }^{\mathrm{b}}$,

${ }^{a}$ Shizuoka Prefectural Research Institute of Agriculture and Forestry, Shizuoka 438-0803, Japan and ${ }^{b}$ Graduate School of Agriculture, Kyoto University, Kyoto

14 Corresponding author. Fax: +81 757536062,

15 E-mail address: WANG@weed.mbox.media.kyoto-u.ac.jp 


\section{Abstract}

17 All living organisms spontaneously generate ultraweak photon emissions, which 18 originate from biochemical reactions in cells. Current research uses the ultraweak 19 photon emission from organisms as a novel indicator in nondestructive analyses of an 20 organisms living state. This study indicates that ultraweak photon emissions from 21 Monochoria vaginalis are different between resistant biotypes (R) to sulfonulurea (SU) 22 and susceptible biotypes (S). In SU-R biotypes, distinct increases in photon emissions 23 were observed, but there was little increase in SU-S biotypes. In addition, photon 24 emissions from the resistant biotypes of $M$. vaginalis were suppressed by treatment 25 with P450 inhibitors. This suggests that cytochrome P450 monooxygenase, which 26 plays a crucial role in the metabolic detoxification of SUs, could be associated with the 27 generation of ultraweak photon emissions. Ultraweak photon emissions have a 28 potential use in a novel diagnosis system as an indicator in a nondestructive testing of 29 weeds resistant to SUs.

30 Keywords: Cytochrome P450 monooxygenase; Herbicide resistance, Monochoria 31 vaginalis, Photon counter, Sulfonylurea, Ultraweak photon emission 


\section{Introduction}

Acetolactate synthase (ALS) ${ }^{1}$ inhibitor-resistant (R) biotypes of weeds have increased worldwide [1], and to date, the biotypes of 95 weed species have been reported [2]. Sulfonylurea (SU) herbicides are one of the most potent ALS-inhibiting herbicides used worldwide. An SU-R weed biotype was first reported in 1990 [3, 4], and since then, the number of SU-R weed species has increased dramatically [2].

Visual effects of SU herbicides on weeds develop slowly because their target site is the inhibition of the enzyme that catalyzes the biosynthesis of branched chain amino acids [5]; hence, it takes a long time to judge the effect of SU herbicides visually. In order to construct a control strategy for SU-R weed biotypes, a rapid identification system is essential. Gerwick et al. [6] developed an in vivo assay method to identify SU-R biotypes, which depended on ALS activity. The method has been applied to several weed species [7-10]. In addition, a simpler method was proposed based on observations of growth inhibition of roots or shoots treated with SU herbicides [11-13].

Among such methods, it was reported the potential of ultraweak photon emission as a novel indicator of resistance biotypes[14]. Ultraweak photon emissions, commonly referred to as biophotons, are very weak light emissions from biological systems with intensities in the order of a few to hundreds of photons per second per square centimeter of surface area, and an almost continuous spectrum within the optical range of at least 200-800 nm [15,16]. Current research makes it clear that ultraweak photon emissions are associated with physiological conditions and can be an effective indicator in nondestructive analyses of organisms in a living state $[17,18]$. In our previous work, it was demonstrated that the intensity of ultraweak photon emission from Scripus juncoides treated with SU herbicides was different between the SU-resistant biotype and susceptible biotypes, i.e. the biophoton emission intensity after SU treatment was higher in the resistant biotypes than in the susceptible biotypes [14]. However, it is unknown whether this photon emission from resistant biotypes was found in other weed species.

Although the precise molecular mechanism underlying ultraweak photon emission has not been fully clarified, it has been suggested that ultraweak photon emission occurs as a result of fluorescent substances, including unsaturated fatty acids, nucleic acids, amino acids, and polyphenols, being peroxidized and excited by reactive oxygen

\footnotetext{
${ }^{1}$ Abbreviations: ALS, acetolactate synthase; BSM, bensulfuron-methyl; DMF, $N^{\prime} N^{\prime}$-dimethyl
} formamide; P450, cytochrome P450 monooxygenase; R, resistant; S, susceptible; SU, sulfonylurea. 
66

67

68

69

species; by energy transfer from excited carbonyl or other substances to luminescence substances. The detoxification metabolism includes oxidation-reduction reactions. In fact, it was reported that an extremely strong increase in biophoton intensity is observed when rice and barnyard grass, which has tolerance by detoxification metabolism, are treated with SU herbicide [19]. Furthermore, these photon emissions from rice and barnyard grass were suppressed when the leaf segments were treated with cytochrome P450 monooxygenase (P450) inhibitors, and it is suggested that this generation of emission is associated with $\mathrm{P} 450$, which is an enzyme involved in oxidative metabolism [20-24].

Therefore we hypothesize ultraweak photon emission from weed treated with SU might be associated with detoxification metabolism with P450. In this study, we studied the difference in ultraweak photon emissions between SU-R and -S biotypes of Monochoria vaginalis, and effect of P450 inhibitors on the ultraweak photon emissions from M. vaginalis.

\section{Materials and methods}

\subsection{Plant samples}

Four SU-R and four SU-S biotypes of $M$. vaginalis were collected from rice paddy fields in Japan and identified by a root bioassay using an 'Instant test-in-office kit' (DuPont Japan Ltd., Tokyo, Japan) ${ }^{2}$. A mutation site of an ALS gene was checked by restriction analysis and direct sequencing. Kamituneyoshi-R has a mutation with a Pro197 to Ser in ALS3 and Zennoji-R has a mutation with a Pro197 to Ser in ALS1, respectively [25]. Wakamiya-R has a mutation with a Asp376 to Glu in ALS1 [26], and Keisen-R has a mutation with a Ala205 to Val in ALS1 (Imaizumi, unpublished). Their self-pollinated seeds were sown and cultivated in 1/5,000a Wagner pots at Kyoto University, Kyoto, Japan.

\subsection{Chemicals}

An SU herbicide, bensulfuron-methyl (BSM) [methyl- $\alpha$-(4,6-dimethoxypyrimidin -2-yl-carbamoylsulfamoyl)-o-toluate] (DuPont Japan Ltd.), most commonly used in paddy fields in Japan, was used in this study.

\footnotetext{
2 DuPont Japan Ltd., 2-11-1 Nagata-Cho, Chiyoda-Ku, Tokyo 100-6111, Japan.
} 
Sulfometuro-metyl was purchased from the Sigma Aldrich Japan Co. (Tokyo, Japan) ${ }^{3}$.The P450 inhibitors, malathion (Wako Pure Chemical Industries, Ltd., Osaka, Japan) ${ }^{4}$ were dissolved in $N^{\prime} N^{\prime}$-dimethyl formamide (DMF; Wako Pure Chemical Co., 99 Ltd., Osaka, Japan) ${ }^{5}$ as 100 -fold stock solutions. We confirmed that the BSM solution,

100 P450 inhibitors and distilled water never generated photon emissions by themselves.

101

102

103

104

105

106

107

108

109

110

111

112

113

114

115

116

117

118

119

120

121

122

123

\subsection{Apparatus for ultraweak photon emission measurements}

Ultraweak photon emissions were detected with a photon counting method using a photon counter PCX-100 (Hamamatsu Photonics K.K., Hamamatsu, Japan) ${ }^{6}$. The PCX-100 is equipped with an R329 photomultiplier tube, which provides a spectral response from 240 to $630 \mathrm{~nm}$. The usable area for measurement of Petri dishes is 16.7 $\mathrm{cm}^{2}$. The photomultiplier moves onto 16 samples, and ultraweak photon emissions from samples were measured in rotation per $10 \mathrm{~s}$ at appropriate times.

\subsection{Measurements of ultraweak photon emissions from sulfonylurea-resistant and -susceptible biotypes in $\mathrm{M}$. vaginalis}

A leaf of each plant was cut into a $5 \mathrm{~mm}$ square, and $0.3 \mathrm{~g}$ of these segments were set in Petri dishes (60 mm diameter), to which $3 \mathrm{ml}$ of a $100 \mathrm{ppm}$ BSM solution or distilled water (control) was added. Samples were set immediately in the photon counter PCX-100 after treatment, and the ultraweak photon emissions from each sample were continuously measured every $10 \mathrm{~s}$ for $42 \mathrm{~h}$. The data from 24 to $40 \mathrm{~h}$, in which ultraweak photon emissions were stabilized, were analyzed. All experiments were performed in triplicate.

2.5. Effect of $\mathrm{P} 450$ monooxygenase inhibitors on ultraweak photon emissions from $\mathrm{M}$. vaginalis

Four biotypes, two SU-R (Keisen and Zennouji) and two SU-S (Maizuru and Wakamiya), were used. Leaf segments (0.3 g) of $M$. vaginalis were set in Petri dishes (60 mm diameter), to which $3 \mathrm{ml}$ of a 100 ppm BSM solution or P450 inhibitors dissolved in distilled water was added. The concentrations of inhibitors used for analysis were and $150 \mathrm{nM}$ of malathion. They were set in the photon counter PCX-100,

\footnotetext{
3 Sigma Aldrich Japan Co., 2-2-24, Higashishinagawa, Shinagawa-Ku, Tokyo, 142-0002, Japan.

${ }^{4}$ Wako Pure Chemical Industries, 1-2, Doshomachi 3-Chome, Chuo-Ku, Osaka 540-8605, Japan.

${ }^{5}$ Wako Pure Chemical Industries, 1-2, Doshomachi 3-Chome, Chuo-Ku, Osaka 540-8605, Japan.

6 Hamamatsu Photonics K. K., 5000 Hiraguchi, Hamamatsu City, Shizuoka 435-8558, Japan.
} 
124 and the ultraweak photon emissions from each sample were continuously measured 125 every $10 \mathrm{~s}$ for $48 \mathrm{~h}$. The data from 24 to $40 \mathrm{~h}$ were analyzed. All experiments were 126 performed in triplicate.

\section{3. Results}

128 3.1. Ultraweak photon emissions from sulfonylurea-resistant and -susceptible biotypes 129 in M. vaginalis

130 Table 1 shows the amino acids of ALS at the Pro197, Ala205 and Asp376 sites encoded by ALS genes in the biotypes used. Four SU-R biotypes have different mutation site and amino acid substitution, respectively. Figure 1 shows the increases in ultraweak photon emissions from the various biotypes of $M$. vaginalis. In the four $\mathrm{R}$ biotypes, distinct increases in intensity of photon emissions were observed when BSM was applied compared with the water control. This higher increase was irrespective of differences in the mutation sites of the ALS genes. In contrast, increases in photon emissions in the four $\mathrm{S}$ biotypes were less than that in the four R biotypes. In particular, there was little difference in photon emissions between BSM application and the water control in Maizuru-S.

3.2. Effect of $P 450$ monooxygenase inhibitors on ultraweak photon emissions from $\mathrm{M}$. vaginalis

To identify the effect of P450 inhibitors on ultraweak photon emissions from $M$. vaginalis treated with BSM, pharmacological analyses were carried out using the P450 inhibitors malathion. Application of malathion alone had little effect on the ultraweak photon emissions of $M$. vaginalis (data not shown).

Figure 2 shows the increases in ultraweak photon emissions from $M$. vaginalis treated with BSM and the P450 inhibitors, and malathion. In two R biotypes, and malathion decreased ultraweak photon emissions from $M$. vaginalis treated with BSM. The increase of ultraweak photon emissions with BSM treatment in the Keisen-R biotype was suppressed by $56 \%$ with malathion. Those in Zennouji-R were suppressed by $84 \%$ with malathion. In contrast, in two susceptible biotypes, there was no definite inhibition of intensity of ultraweak photon emissions with BSM treatment.

\section{Discussion}

Ultraweak photon emissions were initially reported by Colli et al. [27]. Since then, there have been several reports regarding the use of ultraweak photon emission 
157

158

159

160

161

162

163

164

165

166

167

168

169

170

171

172

173

174

175

176

177

178

179

180

181

182

183

184

185

186

187

188

189

190

191

192

intensity as a practical indicator incorporating simplicity and rapidity to investigate the physiological state of plants [28-32]. However, a precise mechanism underlying ultraweak photon emissions has not been fully revealed.

In this study, we demonstrated that BSM treatment induced leaf segments of $M$. vaginalis to generate ultraweak photon emissions, and increases of photon emissions were higher in SU-R biotypes than in SU-S biotypes. In addition, the difference of photon emission between $\mathrm{R}$ and $\mathrm{S}$ biotypes could be indicated in spite of variation of mutation site and amino acid. It is known that target site resistance to SU herbicides has caused by substitution of one of four amino acids (Pro197, Ala205, Asp376, Trp574) [33]. Our data in this study indicate increase of ultraweak photon emissions was observed in M. vaginalis with substitution in Pro197, Ala205, and Asp376. Furthermore, our previous work indicated increase of ultraweak photon emissions was observed in Scirpus juncoides with substitution in Pro197 and Trp574 [18]. Namely, increase of photon emission could be observed in all of four mutation sites. On the other hands, this suggests that increases of photon emissions in SU-R biotypes are not species specific, but rather a general phenomenon, and it further supports our hypothesis that ultraweak photon emissions might be a novel indicator for identifying SU-R biotypes.

To make decisions for the timely management of SU-R weed biotypes, identifying resistance is important. Therefore, several diagnoses of resistant biotypes of $M$. vaginalis have been developed. Yong et al. [34] examined several various techniques to detect SU-R biotypes, and proposed in vitro assays as a simple and quick method. Also, Hamamura et al. [35] and Ohno [36] proposed whole-plant bioassays as a simpler method based on observations of growth inhibition. Ultraweak photon emissions have gained considerable attention in several study fields as an extremely cheap, rapid, simple and reliable indicator with which to investigate the physiological states of plants. We propose ultraweak photon emissions as a possible novel diagnosis system for $\mathrm{R}$ weeds if the generation of ultraweak photon emissions is correctly associated with herbicidal selectivity.

At present, the mechanism of generation of photon emissions depending on BSM treatment is not fully clear. In the previous study, however, we reported that the ultraweak photon emissions from rice and barnyard grass, which are resistant to SUs, were suppressed by P450 inhibitor treatments [19]. Therefore, we hypothesize that P450 might influence ultraweak photon emission in SU-R biotypes of M. vaginalis.

The results presented in this study indicate that photon emissions from $\mathrm{R}$ biotypes of $M$. vaginalis were suppressed by P450 inhibitor treatments. Although a precise 
193

194

195

196

197

198

199

200

201

202

203

204

205

206

207

208

209

210

211

212

213

214

215

216

217

218

219

220

221

222

223

224

mechanism underlying photon emissions remains largely unknown, our data showed the possibility that P450, which play a crucial role in the metabolic detoxification of SUs, may be associated with the generation of ultraweak photon emissions caused by SUs. It is well known that enzyme reactions such as oxidation by lypoxygenase and peroxidase are a source of photon emissions [37-39]. Therefore, it is possible that enzyme reactions in P450 inhibitors are directly related to photon emissions.

\section{Acknowledgments}

This study was supported by a research grant from Shizuoka Prefecture, Japan. BSM was kindly supplied by Kumiai Chemical Industry Co. Ltd. (Tokyo, Japan).

\section{References}

[1] L.L. Saari, J.C. Cotterman, D.C. Thill, Resistance to acetolactate synthase inhibiting herbicides, in: S.B. Powles, J.A.M. Holtum (Eds.), Herbicide Resistance in Plants: Biology and Biochemistry, CRC Press, Boca Raton, 1994, pp. 83-138.

[2] I. Heap, International survey of herbicide-resistant weeds. Available at http://www.weedscience.org/in. Accessed November 5, 2007.

[3] C.A. Mallory-Smith, D.C. Thill, M.J. Dial, Identification of sulfonylurea herbicide-resistance in prickly lettuce (Lactuca serriola), Weed Technol. 4 (1990) 163-168.

[4] M.M. Primiani, J.C. Cotterman, L.L. Saari, Resistance of Kochia (Kochia scoparia) to sulfonylurea and imidazolinone herbicides, Weed Technol. 41 (1990) 169-172.

[5] M.S. Stidham, Herbicides that inhibit acetohydroxyacid synthase, Weed Sci. 39 (1991) 425-434.

[6] B.C. Gerwick, L.C. Mireles, R.J. Eilers, Rapid diagnosis of ALS/AHAS-resistant weeds, Weed Technol. 7 (1993) 519-524.

[7] S.T. Lovell, L.M. Wax, D.M. Simpson, M. McGlamery, Using the in vivo acetolactate synthase (ALS) assay for identifying herbicide-resistant weeds, Weed Technol. 10 (1996) 936-942.

[8] D.M. Simpson, E.W. Stoller, L.M. Wax, An in vivo acetolactate synthase assay, Weed Technol. 9 (1995) 17-22. 
225

226

227

228

229

230

231

232

233

234

235

236

237

238

239

240

241

242

243

244

245

246

247

248

249

250

251

252

253

254

255

256

257

258

[9] A. Uchino, Rapid diagnosis of sulfonylurea-resistant biotypes based on acetolactate synthase activity in the lowland weeds of Japan (in Japanese), J. Weed Sci. Technol. 47 (2002) 197-201.

[10] A. Uchino, H. Watanabe, G.-X. Wang, K. Itoh, Light requirement in rapid diagnosis of sulfonylurea-resistant weeds of Lindernia spp. (Scrophulariaceae), Weed Technol. 13 (1999) 680-684.

[11] P. Boutsalis, Syngenta quick-test: A rapid whole-plant test for herbicide resistance, Weed Technol. 15 (2001) 257-263.

[12] K. Hamamura, T. Muraoka, J. Hashimoto, A. Tsuruya, H. Takahashi, T. Takeshita, K. Noritake, Identification of sulfonylurea-resistant biotypes of paddy field weeds using a novel method based on their rooting responses, Weed Biol. Manag. 3 (2003) 242-246.

[13] S. Ohno, K. Yanagisawa, R. Hanai, T. Muraoka, Shoot-regeneration method: a simplified method of evaluating sulfonylurea herbicide resistance (in Japanese), J. Weed Sci. Technol. 49 (2004) 277-283.

[14] H. Inagaki, Y. Ishida, A. Uchino, K. Kato, C. Kageyama, H. Iyozumi, and H. Nukui, Difference in ultraweak photon emissions between sulfonylurea-resistant and sulfonylurea -susceptible biotypes of Scirpus juncoides following the application of a sulfonylurea herbicide. Weed Biol. Management. 8 (2008) 78-84.

[15] F. A. Popp, Some essential questions of biophoton research and probable answers. In Recent Advances in Biophoton Research and it's Application (Edited by F. A. Popp, Li, K. H., Gu, Q.), pp. 1-42. World Scientific, Singapore, 1992, pp1-42.

[16] H. Inaba, Ultraweak biophoton imaging and information characterization, In: T. Sueta and T. Okoshi (Eds.), Ultrafast and Ultra-Parallel Optoelectronics. Ohmsha/John Wiley and Sons, Tokyo, 1995, pp. 570-580.

[17] R. Kawabata, T. Miike, M. Uefune, H. Okabe, M. Takagi, S. Kai, Biophoton measurement of herbivore-induced plant responses, Jpn. J. Appl. Entomol. Zool. 48 (2004) 289-296.

[18] M. Kobayashi, B. Devaraj, M. Usa, Y. Tanno, M. Takeda, H. Inaba, Development and applications of new technology for two-dimensional space-time characterization and correlation analysis of ultraweak biophoton information, Frontiers Med. Biol. Engng. 7 (1996) 299-309. 
259

260

261

262

263

264

265

266

267

268

269

270

271

272

273

274

275

276

277

278

279

280

281

282

283

284

285

286

287

288

289

290

291

[19] H. Inagaki, T. Imaizumi, G.-X. Wang, T. Tominaga, K. Kato, H. Iyozumi, H. Nukui, Spontaneous ultraweak photon emission from rice (Oryza sativa L.) and paddy weeds treated with a sulfonylurea herbicide, Pestic. Biochem. Physiol. 89 (2007) 158-162.

[20] S. Shirakura, K. Ito, H. Aizawa, Effect of cytochrome P-450 monooxygenase inhibitors on the rice tolerance of azimsulfuron and bensulfuron-methyl, J. Weed Sci. Tech. 40 (1995) 218-220.

[21] R.P. Donaldson, D.G. Luster, Multiple forms of plant cytochromes P-450, Plant Physiol. 96 (1991) 669-674.

[22] A. Zimmerlin, F. Durst, Aryl hydroxylation of the herbicide diclofop by a wheat cytochrome P-450 monooxygenase, Plant Physiol. 100 (1992) 874-881.

[23] S. Shirakura, K. Ito, A.C. Barefoot, H. Aizawa, K. Ishizuka, Difference in absorption, translocation and metabolism of azimsulfuron between rice (Oryza sativa) and flat sedge (Cyperus serotinus), Weed Res. Jpn. 40 (1995) 299-307.

[24] C.S. Kwon, D. Penner, Response of a chlorsulfuron-resistant biotype of Kochia scoparia to ALS-inhibiting herbicides and piperonyl butoxide, Weed Sci. 43 (1995) 561-565.

[25] T. Ohsako, R. Sugihara, K. Fujimoto, T. Tominaga. Genetic variation of the sulfonylurea- resistant biotypes of Monochoria vaginalis. J. Weed Sci. Tech. 50 (Sup.) (2005) 196-197 (in Japanese).

[26] T. Imaizumi, G.-X. Wang, T Ohsako, T. Tominaga. Genetic diversity of sulfonylurea-resistant and -susceptible Monochoria vaginalis populations in Japan. Weed Res. 48 (2008) 187-196.

[27] L. Colli, U. Facchini, G. Guidotti, R. Dungani, M. Orsenigo, O. Sommariva, Further measurement on the bioluminescence of the seedlings. Experimentia 11 (1955) 479-481.

[28] E. Hideg, M. Kobayashi, H. Inaba, Ultraweak photon emission from dark-adapted leaves and isolated chloroplasts, FEBS Lett. 275 (1990) 121-124.

[29] E. Hideg, H. Inaba, Dark adapted leaves of paraquat-resistant tobacco plants emit less ultraweak light than susceptible ones, Biochem. Biophys. Res. Commun. 178 (1991a) 438-443.

[30] E. Hideg, H. Inaba, Biophoton emission (ultraweak emission) from dark adapted spinach chloroplasts, Photochem. Photobiol. 53 (1991b) 137-142. 
292

293

294

295

296

297

298

299

300

301

302

303

304

305

306

307

308

309

310

311

312

313

314

315

316

317

318

319

[31] H. Iyozumi, K. Kato, C. Kageyama, H. Inagaki, A. Yamaguchi, K. Furuse, K. Baba, H. Tsuchiya, Plant defense activators potentiate the generation of elicitor-responsive photon emission in rice, Physiol. Mol. Plant Pathol. 66 (2005) 68-74.

[32] N. Yoshinaga, K. Kato, C. Kageyama, K. Fujisaki, R. Nishida, N. Mori, Ultraweak photon emission from herbivory-injured maize plants, Naturwissenschaften 93 (2006) 38-41.

[33] P.J. Tranel, T.R. Wright. Resistance of weed to ALS-inhibiting herbicides: what have we learned?, Weed Sci. 50 (2002) 700-712.

[34] I.K. Yong, H.I. Jung, O.D. Kwon, D.J. Lee, R.B. Nilda, J.O. Guh, Rapid diagnosis of resistance to sulfonylurea herbicides in monochoria (Monochoria vaginalis), Weed Sci. 51 (2003) 305-311.

[35] K. Hamamura, T. Muraoka, J. Hashimoto, A. Tsuruya, H. Takahashi, T. Takeshita, and K. Noritake. Identification of sulfonylurea-resistant biotypes of paddy field weeds using a novel method based on their rooting responses. Weed Biol. Manag. 3 (2003) 242-246.

[36] S. Ohno, K. Yanagisawa, R. Hanai, and T. Muraoka. Shoot regeneration method: a simplified method of evaluating sulfonylurea herbicide resistance (in Japanese). J. Weed Sci. Technol. 49 (2004) 277-283.

[37] A. Boveris, E. Cadenas, B. Chance, Low level chemiluminescence of the lipoxygenase reaction, Photobiochem. Photobiophys. 1 (1980) 175-182.

[38] F.B. Abeles, Plant chemiluminescence, Annu. Rev. Plant Physiol. 37 (1986) 49-72.

[39] G.P. Bolwell, L.V. Bindschedler, K.A. Blee, V.S. Butt, D.R. Davies, S.L. Gardner, C. Gerrish, F. Minibayeva, The apoplastic oxidative burst in response to biotic stress in plants: a three-component system, J. Exp. Bot. 53 (2002) 1367-1376. 


\section{Figure Legends}

321 Fig. 1. The increases in ultraweak photon emissions of resistant and susceptible

322 biotypes of Monochoria vaginalis. The increases of photon emissions were the

323 differences in averages during 24-40 h after treatment between the SUs application

324 and water control. Photon emissions were continuously measured with a PCX-100

325 multisample photon counter. Values represent the average of three replications. Bars

326 indicate standard deviations $( \pm \mathrm{SD})$. Different letters indicate a significant difference at

327 the 5\% level according to Tukey’s Studentized Range Test.

328

329 Fig. 2. Effect of P450 inhibitors on ultraweak photon emissions from leaf segments of

330 M. vaginalis treated with SU herbicides. The increases of photon emissions were the

331 averages during $24-40 \mathrm{~h}$ after SU and P450 inhibitor treatment to the water control.

332 Photon emissions were continuously measured with a PCX-100 multisample photon

333 counter. Values represent the average of three replications. Bars indicate standard

334 deviations $( \pm \mathrm{SD})$. * and ** indicate the significant deiierences at $\mathrm{P}<0.05$ and 0.01 ,

335 respectively. 
Fig. 1. --- Inagaki et al. --- $\uparrow$

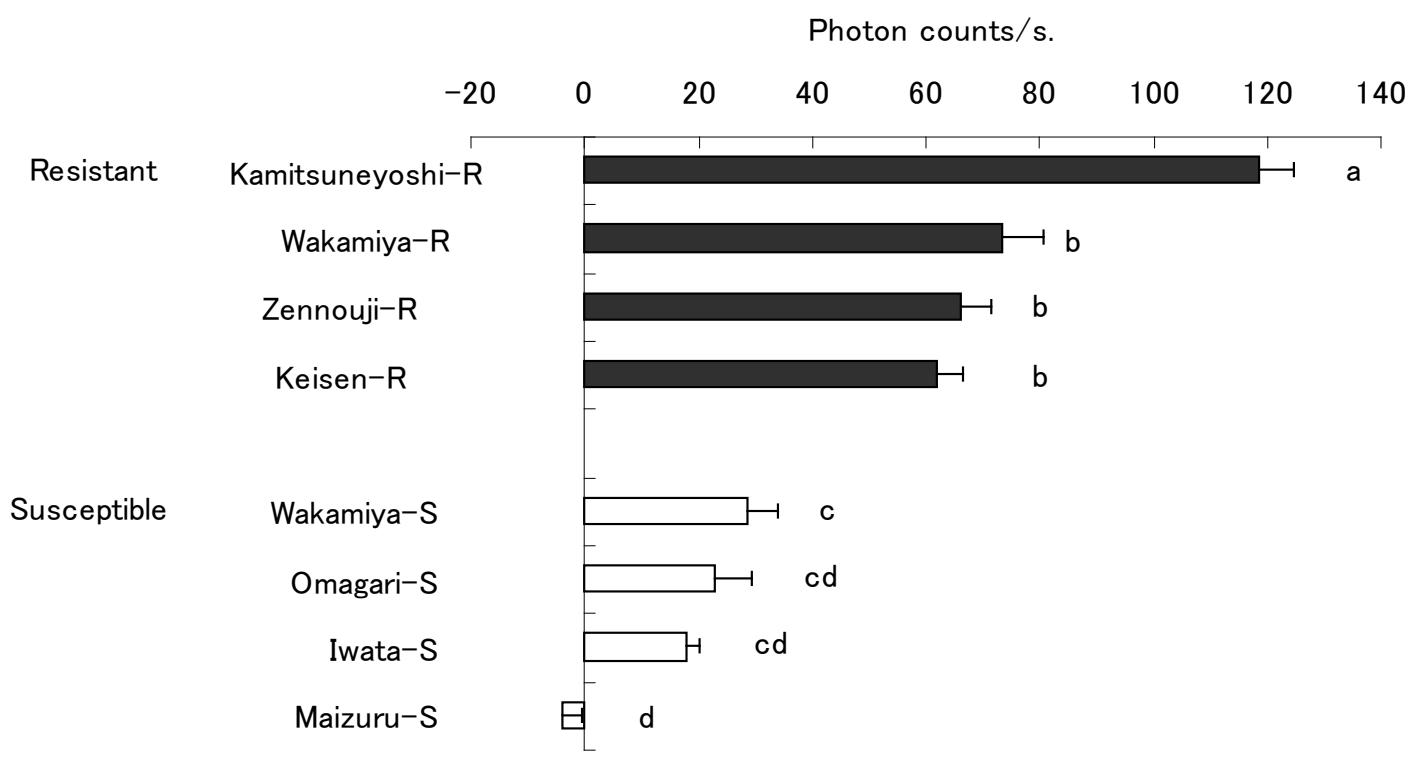


Fig. 2. --- Inagaki et al. --- $\uparrow$
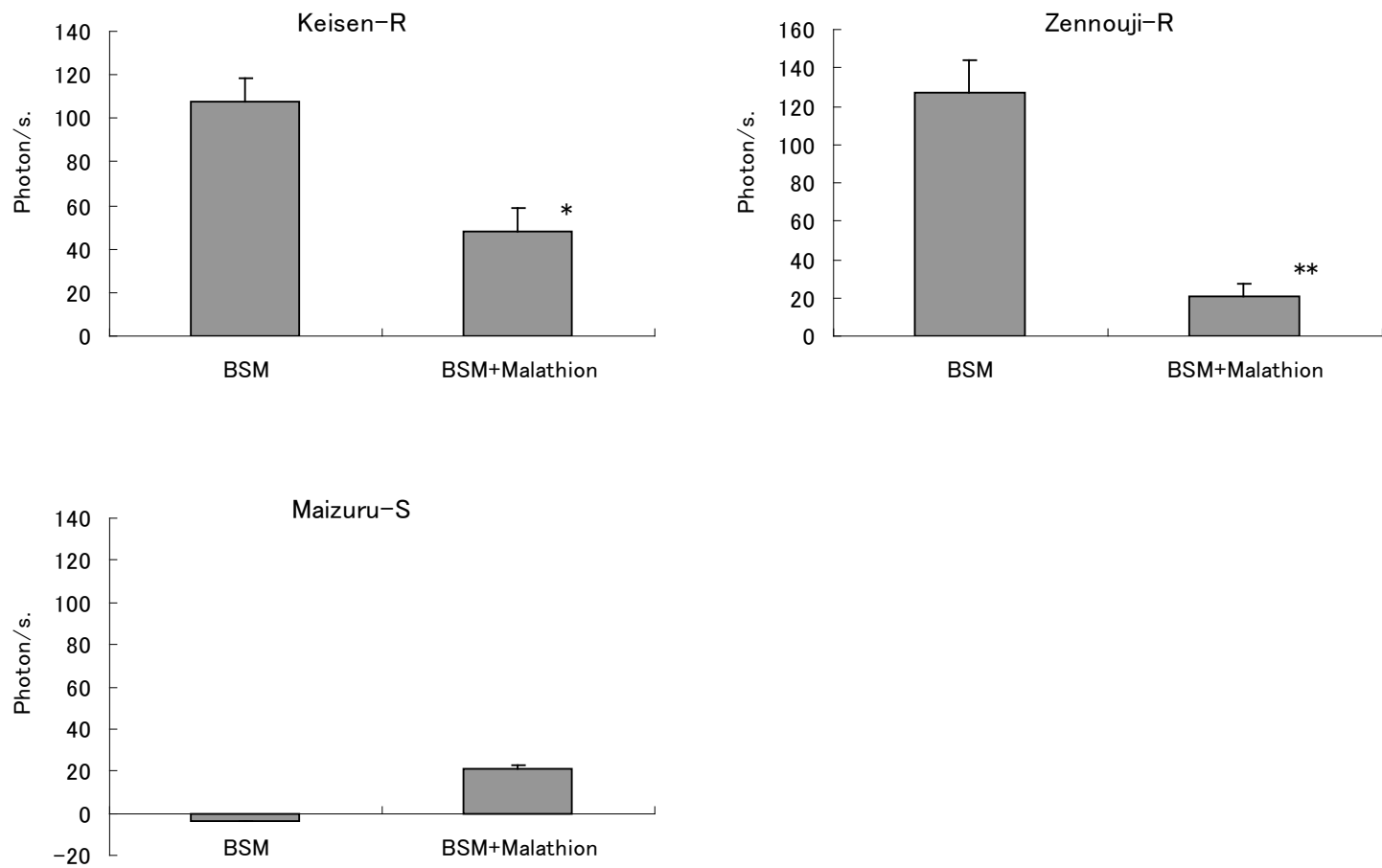